\title{
AA-stacked bilayer square ice between graphene layers
}

\author{
M. Sobrino Fernandez Mario, ${ }^{1}$ M. Neek-Amal, ${ }^{2,}{ }^{*}$ and F. M. Peeters ${ }^{1}$ \\ ${ }^{1}$ Departement Fysica, Universiteit Antwerpen, Groenenborgerlaan 171, B-2020 Antwerpen, Belgium \\ ${ }^{2}$ Department of Physics, Shahid Rajaee Teacher Training University, Lavizan, Tehran 16788, Iran \\ (Received 26 August 2015; revised manuscript received 6 November 2015; published 17 December 2015)
}

\begin{abstract}
Water confined between two graphene layers with a separation of a few $\AA$ forms a layered two-dimensional ice structure. Using large scale molecular dynamics simulations with the adoptable ReaxFF interatomic potential we found that flat monolayer ice with a rhombic-square structure nucleates between the graphene layers which is nonpolar and nonferroelectric. We provide different energetic considerations and H-bonding results that explain the interlayer and intralayer properties of two-dimensional ice. The controversial AA stacking found experimentally [Algara-Siller et al., Nature (London) 519, 443 (2015)] is consistent with our minimum-energy crystal structure of bilayer ice. Furthermore, we predict that an odd number of layers of ice has the same lattice structure as monolayer ice, while an even number of ice layers exhibits the square ice AA stacking of bilayer ice.
\end{abstract}

DOI: 10.1103/PhysRevB.92.245428

PACS number(s): 73.22.Pr, 64.70.Nd, 71.15.-m

\section{INTRODUCTION}

The phase diagram of water and its extraordinary properties have been an interesting topic of research in biology, chemistry, and physics for many decades. Depending on the hydrophobic confinement width several two-dimensional ice structures can be formed [1-4]. Different theoretical methods, e.g., molecular dynamics (MD) simulations using different force fields [1,4], density functional theory [5], and Monte Carlo simulations [6], have been used to study ice formation in the presence of high pressure. In particular, monolayer ice was proposed by Zangi and Mark [1,2] using MD simulations by applying a five site and tetrahedrally coordinated model, i.e., TIP5P. They confined water between two parallel plates and applied a high lateral pressure $\left(P_{l}\right)$ of about 1-2 kbar and found a nonflat monolayer of ice.

Recently, it was found experimentally that confined water exists as a quasi-two-dimensional layer with different properties than those of bulk water $[7,8]$. Graphene, the two-dimensional allotrope of carbon [9], was used in a recent experiment to confine water [8] into monolayer, bilayer, and three layers. Using transmission electron microscopy (TEM) a square lattice structure was observed. The lateral pressure for confining water between two sheets of graphene can be estimated $[8,10,11]$ to be about $1 \mathrm{GPa}$ using the van der Waals (vdW) adhesive energy between two layers which is typically around $20 \mathrm{meV}^{-2}$. This experiment was supported by MD simulations that showed that by increasing the pressure, bilayer square ice (three layer ice) with a lattice constant of $2.82 \AA$ nucleates where the graphene layers are separated by a distance $h=9 \AA$ (11.5 $\AA$ ) [8]. However, the MD simulations failed to reproduce the experimental found AA stacking of bilayer ice. Ab initio calculations found that monolayer ice confined between hydrophobic graphene layers can be rippled or flat, depending on the confinement width and lateral pressure [5]. However, this DFT study is based on a small supercell which therefore missed structures that involve more than four water molecules.

\footnotetext{
*Corresponding author: neekamal@ srttu.edu
}

Using the reactive bond order potential we reveal new physics of confined ice between two graphene layers. We performed annealing MD simulations starting from high temperature, i.e., $400 \mathrm{~K}$ and found the low-temperature minimum-energy configuration, and determined the structure of monolayer, bilayer, and three layer ice. We evaluate the different energy terms, charge distribution, and hydrogen bond strength of confined water. The ReaxFF potential allows for different bond formations and takes into account the polarization of charge within the molecules. The studied systems are found to be all flat, nonpolar, and nonferroelectric where the microscopic structure depends on the number of ice layers. We found a flat structure for confined ice and estimated the vdW energy between two ice layers.

\section{METHOD AND MODEL}

We employed molecular dynamics (MD) simulations using reactive force field (ReaxFF) potentials [12] in the well-known large-scale atomic/molecular massively parallel simulator "LAMMPS" [13]. The ReaxFF potentials used [14] account for possible bond formation and bond dissociation of different bond orders. It also contains Coulomb and van der Waals potentials to describe nonbond interactions between all atoms. One of the main advantages of ReaxFF is that it calculates the polarization of charge within the molecules which is achieved by using electronegativity and hardness parameters based on the electronegativity equalization method and charge equilibration $(\mathrm{QEq})$ methods. Therefore, we believe that ReaxFF is a better candidate to simulate water and the corresponding interaction between water and graphene. Furthermore, the ReaxFF potential allows bond extension/contraction in water as well as angle bending and charge relaxation over each atom. This is in contrast to the traditional force fields for water, e.g., SPC and TIP4P [15] (a rigid planar four-site interaction potential for water) that keep the water molecules rigid during MD simulations.

The computational unit cell contains 34848 carbon atoms and $5700 \times n$ water molecules where $n$ is the number of ice layers. These numbers have been chosen so that the graphene lattice is commensurate with the water lattice when performing simulations with periodic boundaries. To 
(a)

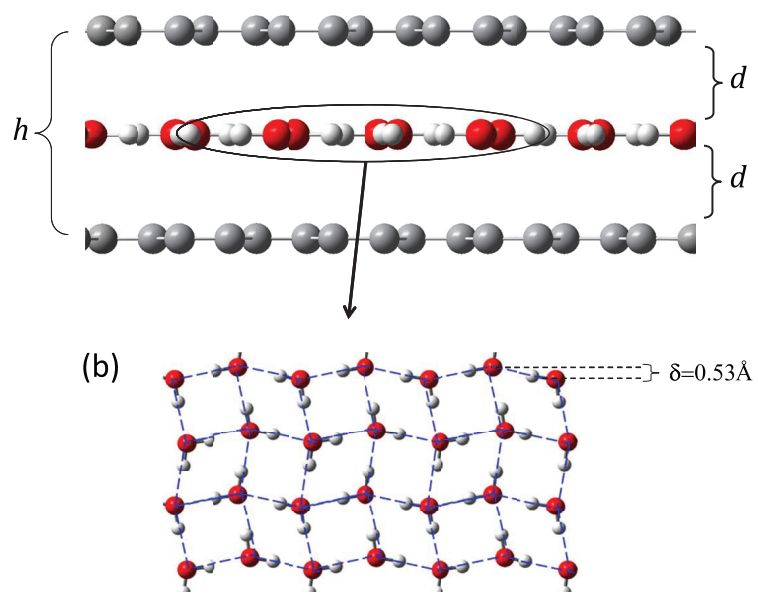

(c)

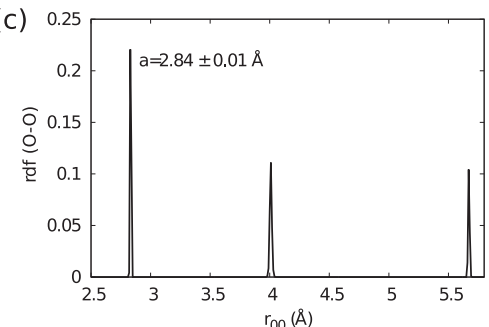

(d)

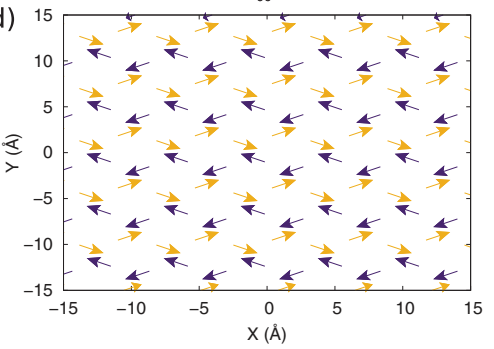

FIG. 1. (Color online) The top (a) and side (b) view of the relaxed monolayer of ice between two graphene layers [red (grey) circles indicate $\mathrm{O}(\mathrm{H})$ atoms]. The corresponding (c) radial distribution function for O-O distances and (d) local dipoles of the individual water molecules.

match two different lattice parameters in a simulation box the following equation should hold: $a_{G E} m=a_{O O} n$, where the lattice constants $a_{O O}=2.83 \AA$ and $a_{G E}=\sqrt{3} / 2 a_{C C}$ and $m$ and $n$ should be integer numbers. Before starting the minimization we do an annealing MD simulation by performing a NPT simulation starting at $400 \mathrm{~K}$ and ending at $0 \mathrm{~K}$, in order to find the true simulation box size and $\mathrm{O}-\mathrm{O}$ distances. Annealing was done using a Nose-Hoover barostat, with a cooling rate of $8 \mathrm{~K} / \mathrm{ps}$ and a damping constant of 0.1 ps. The high starting temperature guarantees that the $\mathrm{O}$ atoms and $\mathrm{H}$ bonds can find their minimum-energy configurations during the slow annealing process. Then, the total energy is minimized using the iterative conjugate gradient (CG) scheme.

\section{A. Monolayer of ice}

We start from a random distribution for the $\mathrm{H}$ bonds of the water molecules which are distributed between two graphene layers in a dense square structure, with $\mathrm{O}-\mathrm{O}$ distance equal to $2.8 \AA$. The initial O-O distance is set to have the experimentally observed density for confined ice but during annealing, which we start from $400 \mathrm{~K}$, the positions are allowed to change. The graphene layers are rigid and separated by a fixed distance of $6.5 \AA$ having $\mathrm{AB}$ stacking. However, the results for the water layer are independent of the exact stacking configuration of the graphene layers. By minimizing the potential energy, we found a flat rhombic-square lattice structure; see Fig. 1. The side view of the minimum-energy configuration is shown in Fig. 1(a) which is a flat monolayer of ice with successive arrangements of square and rhombic building blocks; see Fig. 1(b). If the $\mathrm{O}$ atoms remain in the same plane, the $\mathrm{H}$ atoms should also be in that plane in order to preserve the symmetry. The other possibility would be a buckled (puckered) structure which results in nonflat ice [1] (which was not found with our simulations) [16]. Our results are partially in agreement with $a b$ initio results where Corsetti et al. [5] used nonlocal vdW exchange correlations and scanned both the confinement size and lateral pressure. However, their unit cell (called $\mathrm{Ab} / \mathrm{Cd}$ ) was too small, i.e., it contained only four water molecules, in order to find the aforementioned asymmetry effect. Our found structure for monolayer ice is in agreement with the MD simulation results using the SPC/E model [8]. In fact the TEM result of the recent experiment [8] is not a perfect square lattice layer [17].

The crystalline structure and lattice constant of monolayer ice can be determined from the radial distribution function (RDF); see Fig. 1(c). We found the O-O distance to be $a=2.84 \pm 0.01 \AA$. The obtained lattice constant is in good agreement with the experimental value of $a=2.81 \pm 0.02 \AA$. The angle "H-O-H" is found to be $\theta=106.31 \pm 0.03^{\circ}$ and is identical for all $\mathrm{H}-\mathrm{O}-\mathrm{H}$ bonds. The H-bonding energy is about $-0.16 \mathrm{eV} /$ water $\left(-15.43 \mathrm{~kJ} \mathrm{~mol}^{-1}\right)$ for each water molecule which is in the range of the $\mathrm{H}$-bond energy of bulk ice [18], i.e., $-(13-32) \mathrm{kJ} \mathrm{mol}^{-1}$. It is worth mentioning that the $\mathrm{vdW}$ energy stored in the system is $E_{v d W}=2.43 \mathrm{eV} /$ atom which is positive and the Coulomb energy is $E_{\text {coulomb }}=$ $-0.56 \mathrm{eV} /$ atom which is negative. These numbers show that in dense ice the $\mathrm{O}$ atoms repel each other strongly (note that $a=r_{O O} \approx 2.8 \AA$ lies in the repulsion region of the $\mathrm{vdW}$

TABLE I. Different energy contributions to the minimized-energy configurations for mono (g.ice.g), bilayer (g.ice.ice.g), and trilayer (g.ice.ice.ice.g) water confined between two graphene layers.

\begin{tabular}{lccccccc}
\hline \hline & $\mathrm{H}(\AA)$ & $E_{\text {coulomb }}(\mathrm{eV} /$ atom $)$ & $E_{V d W}(\mathrm{eV} /$ atom $)$ & $E_{H B}(\mathrm{eV} /$ water $)$ & $a=r_{o o}=(\AA)$ & $d(\AA)$ & $c(\AA)$ \\
\hline g.ice.g & 6.5 & -0.56 & 2.43 & -0.16 & $2.84 \pm 0.01$ & $3.25 \pm 0.01$ & 0 \\
g.ice.ice.g & 9 & -0.86 & 2.07 & -0.13 & $2.84 \pm 0.01$ & $2.88 \pm 0.01$ & $3.24 \pm 0.01$ \\
g.ice.ice.ice.g & 11.5 & -0.94 & 1.82 & -0.16 & $2.89 \pm 0.02$ & $2.70 \pm 0.01$ & $3.05 \pm 0.01$ \\
\hline \hline
\end{tabular}




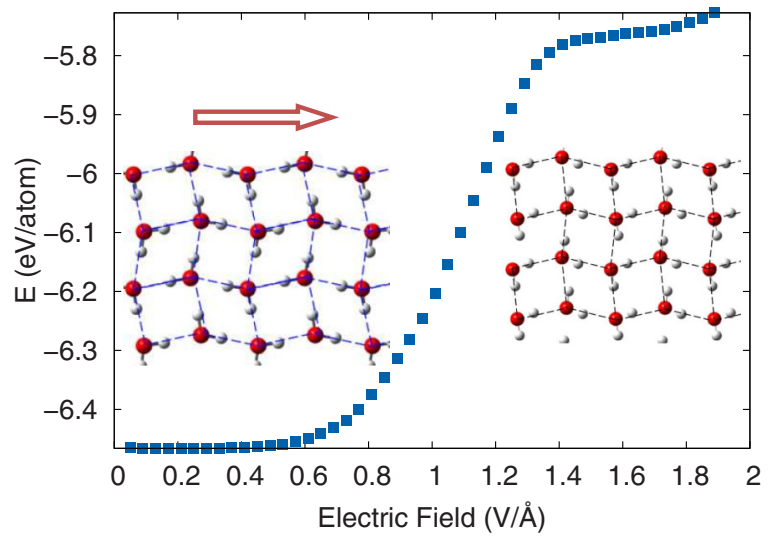

FIG. 2. (Color online) Energy of an ice monolayer relaxed between two graphene layers when applying an in-plane electric field along the direction of the arrow (left inset). Note the change of the $\mathrm{H}$-bond orientation (right inset).

energy function [19]). In Table I, we list all relevant quantities for the different studied systems.

The corresponding local dipoles of the water molecules in the minimum-energy configuration are shown by the arrows in Fig. 1(d). Interestingly, the net dipole is zero and the system is nonferroelectric which is in agreement with ab initio results [5] and is in disagreement with the TIP5P model prediction [20]. We were able to deform the $\mathrm{H}$-bond orientations using an in-plane electric field of about $1 \mathrm{~V} / \AA$ (see Fig. 2). As shown in Fig. 2, by increasing the electric field (along the direction of the shown arrow in the inset of Fig. 2) from zero to around $|E| \simeq 1$ $\mathrm{V} / \AA$ we find a structural transition from the minimum-energy configuration with zero dipole moment to a new higher energy configuration with a net nonzero dipole moment, i.e., the local dipoles of the water molecules rotate in the direction of the electric field (see right inset of Fig. 2).

For nonpolar flat monolayer ice there are many possible structures. We made a detailed investigation of them and present the results of one of the most relevant ones. We performed additional MD annealing and minimization by starting from an initial configuration where the $\mathrm{H}$ bonds are randomly oriented in three dimensions but have the same orientation for each water molecule. We found that the minimum-energy configuration is a flat and polar structure which is shown in Fig. 3. The potential energy of this structure is $10 \mathrm{meV} /$ atom higher than the nonpolar structure (Fig. 1). The vdW, Coulomb, and H-bond energy for the polar monolayer shown in Fig. 3 are found to be $E_{\text {coulomb }}=-0.55 \mathrm{eV} /$ atom, $E_{v d W}=$ $2.48 \mathrm{eV} /$ atom, and $E_{H B}=-0.14 \mathrm{eV} /$ water respectively. The $\mathrm{O}-\mathrm{O}$ distance is $a=2.88 \pm 0.02 \AA$ and the $\mathrm{H}-\mathrm{O}-\mathrm{H}$ angle equals $\theta=106.6 \pm+0.1^{\circ}$. We also performed simulations for the other possible polar structures proposed by Corsetti et al. [5] and found that all of them have higher energy than the one shown in Fig. 1.

\section{B. Bilayer square ice}

Motivated by the experimental work of Algara-Siller et al. [8] a bilayer of ice confined between two rigid graphene layers separated by $h=9 \AA$ was investigated. The minimum-energy
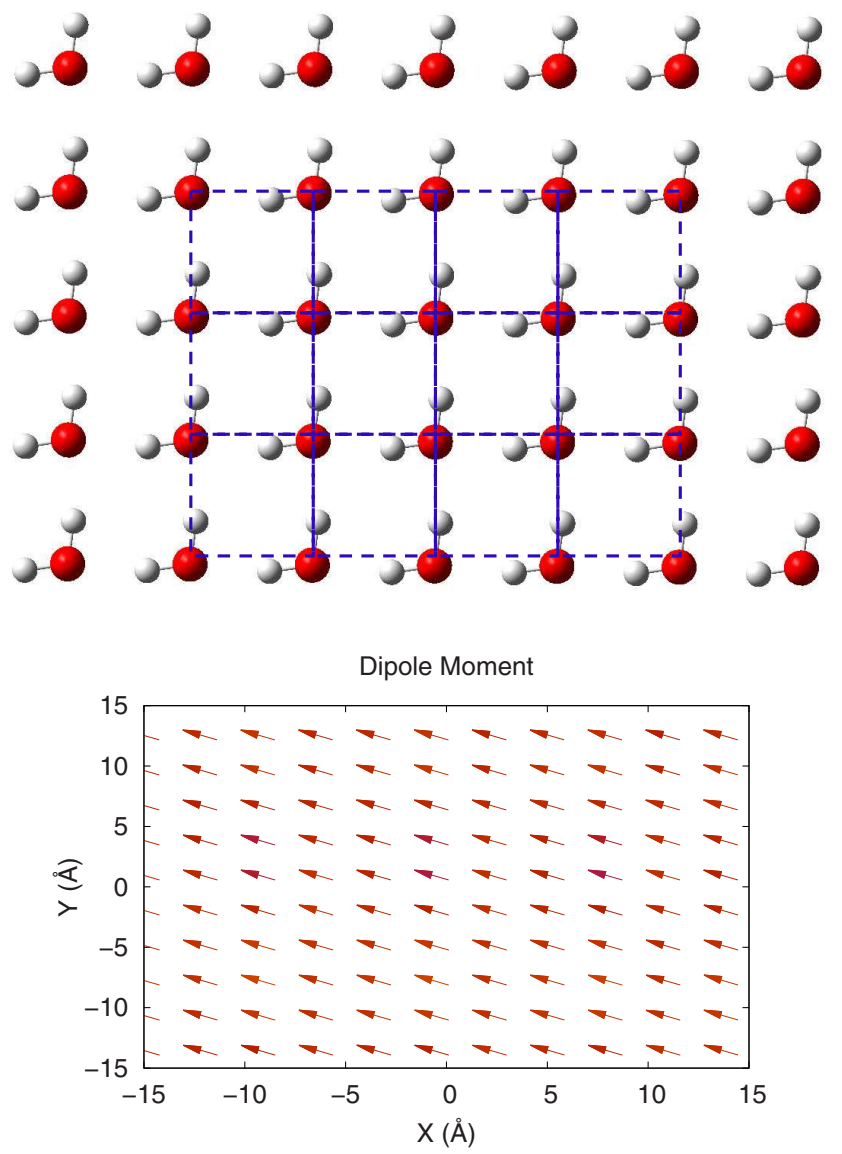

FIG. 3. (Color online) (a) A polar lattice of monolayer ice confined between two graphene layers which has about $10 \mathrm{meV} /$ atom higher energy than the nonpolar structure. (b) The corresponding local dipoles.

structure is shown in Figs. 4(a) and 4(c). Surprisingly, also in this case the layers are flat [see Fig. 4(a)] and each has a perfect square lattice for the $\mathrm{O}$ atoms [see Fig. 4(c)]. Using the radial distribution function for each layer we found the lattice constant to be $a=2.84 \pm 0.01 \AA$. The radial distribution function for each ice layer is presented in Fig. 4(b) and are identical. The obtained angle $\mathrm{H}-\mathrm{O}-\mathrm{H}$ is found to be $\theta=106.15 \pm+0.02^{\circ}$. Each layer has a net dipole that is in the opposite direction with respect to the other layer. The latter makes the bilayer of ice nonpolar and nonferroelectric. The interlayer distance between the ice layers is found to be $c=3.24 \pm 0.01 \AA$ which is in disagreement with the SPC/E model which obtained $a \cong c$ [8]. Our finding for " $c$ " is in the range of vdW adhesion between two ordinary neutral layers, e.g., the two graphene layers. The H-bonding, vdW, and the Coulomb energy are $-0.13 \mathrm{eV} /$ water, $2.07 \mathrm{eV} /$ atom, and $-0.86 \mathrm{eV} /$ atom, respectively. It is interesting to note that the vdW energy here is lower than that of a single layer of ice which is due to the extra adhesion between the two ice layers. Therefore we can estimate the vdW energy stored between the ice layers as $-0.41 \mathrm{eV} /$ atom. This energetic analysis gives important insights about the physics of confined water between two graphene layers.

The stacking of the two ice layers is not (perfect) AA stacking, i.e., the bottom layer has an in-plane shift of about $1.2 \AA$ 
(a)

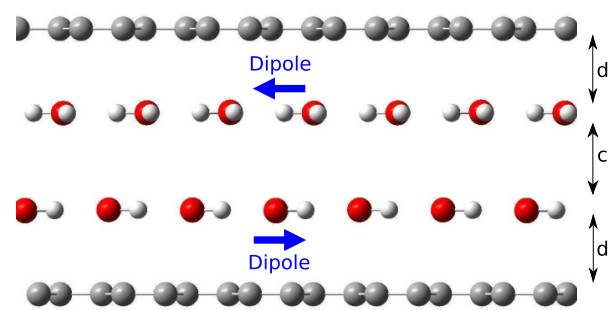

(b)

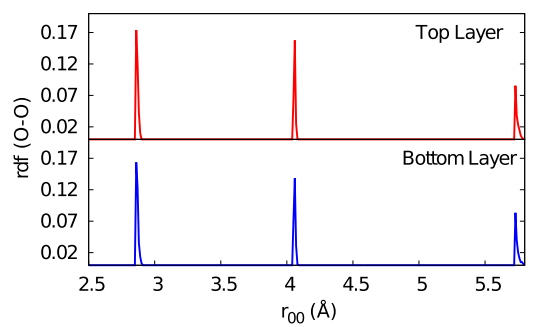

(c) Top layer

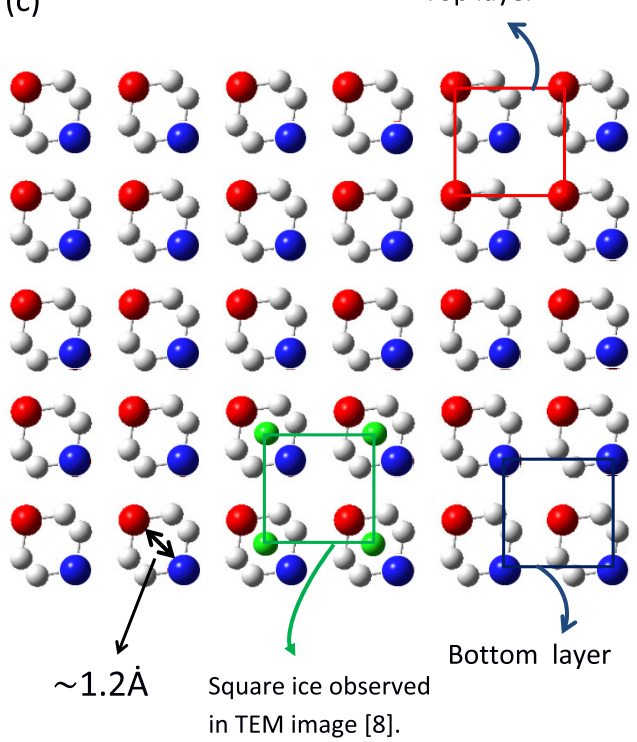

FIG. 4. (Color online) Relaxed bilayer ice between two graphene layers are shown in (a) side and (c) top view. In (b) the corresponding radial distribution function for O-O distances is shown. In the top view (c), the green dots refer to the TEM experiment [8]. The in-plane shift between top and bottom layers is about $1.2 \AA$.

[shown by the arrow in Fig. 4(c)] with respect to the top layer. The displacement of the $\mathrm{O}$ atoms with respect to each other is due to the fact that the $\mathrm{O}$ atoms are negatively charged and thus repel each other. Please note that the SPC/E model [8] predicts $\mathrm{AB}$ stacking for bilayer ice confined between two graphene layers. However, the TEM images in the recent experiment [8], which are shown by green circles in the bottom right part of Fig. 4(c), can be considered as the averaged positions of oxygen atoms in the top (red dots) and bottom layer (blue dots) of our results. We believe that the blue and red circles in reality vibrate along the black arrow shown in Fig. 4(c) resulting in a time averaged AA stacking in square ice. In order to investigate the importance of the interaction between ice and graphene and to present an independent test, we performed an additional MD minimization. We minimized the potential energy of monolayer ice with randomly distributed $\mathrm{H}$ bonds (even out of plane) over a single layer of graphene at an initial distance of 3.0 А. The minimum-energy configuration of ice is similar to Fig. 1 with the distance between graphene and ice of $2.90 \AA$. This shows that the interaction between graphene and an ice layer is stronger than the interaction between two ice layers, which are separated by $c=3.24 \AA$ and the interaction between the two graphene layers even if they are at a distance of $9 \AA$.

\section{Trilayer ice}

Finally, we turn our attention to the stacking and microscopic structure of confined three layer ice. By fixing two graphene layers at a distance $h=11 \AA$ and performing annealing MD simulations, we found that each layer of the three layer ice is nonpolar with a microscopic structure similar to that of monolayer ice [see Fig. 1(b)]. The ice-graphene distance is found to be $d=2.70 \AA \pm 0.01$ and the distance between each of the ice layers is $c=3.05 \stackrel{\AA}{\AA} \pm 0.01$. The vdW, Coulomb, and H-bonding energy are $E_{\text {coulomb }}=-0.94 \mathrm{eV} /$ atom, $E_{v d W}=$ $1.82 \mathrm{eV} /$ atom, and $E_{H B}=-0.16 \mathrm{eV} /$ water respectively.
We found that it is energetically unfavorable to have an ice layer with a net dipole moment next to one with zero net polar moment. Therefore, we conclude that for an odd number of ice layers each layer has the structure of confined monolayer ice (each of them with a zero net dipole moment). However a system with an even number of ice layers confined between graphene will be comprised of pairs of layers where each pair has a structure like bilayer ice (Fig. 5).

\section{DISCUSSION AND CONCLUSIONS}

In ordinary water the average distance between oxygen atoms is about $2.82 \AA$ [18] and for each water molecule two hydrogen bonds ( $\mathrm{H}$ bonds) are randomly oriented resulting in an irregular network. The $\mathrm{H}$ bonds in liquid water have a lifetime of about 1-20 ps. Continuously bond formation and bond breaking takes place. The $\mathrm{H}$ bonds are stronger in hexagonal bulk ice (ordinary ice) though the O-O distance is still $2.82 \AA$ but each water molecule takes part in four tetrahedrally arranged $\mathrm{H}$ bonds [18], i.e., forming a regular network with more room between the molecules yielding a lower density. Moreover, the strength and orientation of the $\mathrm{H}$ bonds change when high pressure is applied. When water is confined between hydrophobic walls (here two graphene layers) in the presence of lateral pressure of about $1 \mathrm{GPa}$, monolayer ice is formed with an O-O distance of about $2.83 \AA$ [8]. Therefore, the spatial orientation of the $\mathrm{H}$-bonds and their strength and rearrangement is a key factor which determines the structure of water and the different phases of ice in confined monolayer and few layer ice.

In typical three-dimensional (3D) ice structures, e.g., ice $I_{h}$, the electrostatic attraction between $\mathrm{H}$ and $\mathrm{O}$ atoms dominates the repulsion between the oxygens. However, in a dense monolayer (and few layer) ice, the electrostatic energy is not large enough to cancel the repulsive vdW energy and thus an external pressure is needed to keep the system stable. In 

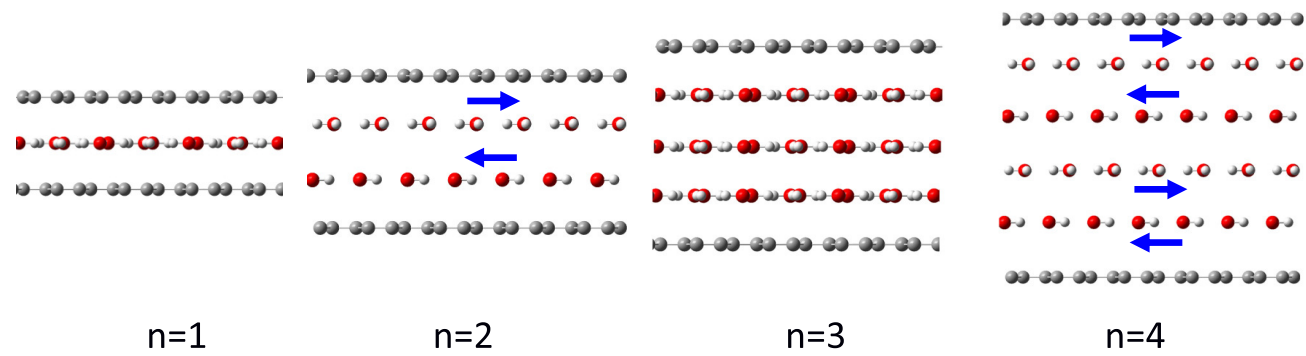

FIG. 5. (Color online) Multilayer ice confined between graphene layers. The arrows indicate the net dipole moment in each layer.

fact, the high lateral pressure applied on confined water at room temperature results in monolayer ice. These conditions are equivalent to the water phase close to zero $\mathrm{K}$ (i.e., ice) but now at about 1 bar (or zero) as we studied. The multiple configurations of ice at high pressures that meet the rules of absolute zero temperature amounts to randomness, or in other words, entropy which is called residence entropy. Therefore the ground-state configuration of water either at zero temperature or high pressures strongly depends on the experimental procedure and details. This might be the reason for the observation of several different crystal structures as reported by different groups for confined ice using various methods $[5,8,20]$. The large degree of freedom for the hydrogen bond strength and its orientation gives the possibility to have several ice structures, either when the system is subjected to high pressure or is kept close to zero temperatures. This large degree of freedom is reduced when using the rigid model in MD simulations which may result in incorrect lattice structures. In our case, however, we find a layered structure which at zero temperature is an entropyless structure. Three-dimensional water ice is the first material for which residual entropy was proposed by Pauling [21]. By using MD simulations we showed that the Pauling proposal can be broken for a flat layer of ice.

For confined monolayer ice with a flat structure and zero net dipole moment the $\mathrm{H}$ bonds should lie in the same plane. However, flat ice layers in nonpolar bilayer of ice nucleates as a square lattice with an almost AA stacking. We predict that an odd (even) number of ice layers are stacks of monolayer (bilayer) ice. We found that the interaction between ice layers is weaker than that between ice and graphene which results in a shorter distance between ice and graphene.

\section{ACKNOWLEDGMENTS}

This work was supported by the ESF-Eurographene project CONGRAN, and the Flemish Science Foundation (FWO-V1).
[1] R. Zangi and A. E. Mark, Phys. Rev. Lett. 91, 025502 (2003).

[2] R. Zangi and A. E. Mark, J. Chem. Phys 120, 7123 (2004).

[3] J. Bai, C. Austen Angell, and X. Cheng Zeng, Proc. Natl. Acad. Sci. USA 107, 5718 (2010).

[4] W-H. Zhao, L. Wang, J. Bai, L-F. Yuan, J. Yang, and X. Cheng Zeng, Acc. Chem. Res. 47, 2505 (2014).

[5] F. Corsetti, P. Matthews, and E. Artacho, arXiv:1502.03750.

[6] C. Vega, C. McBride, E. Sanz, and J. L. F. Abascal, Phys. Chem. Chem. Phys. 7, 1450 (2005).

[7] M. Ghosh, L. Pradipkanti, V. Rai, D. K. Satapathy, P. Vayalamkuzhi, and M. Jaiswa, Appl. Phys. Lett. 106, 241902 (2015).

[8] G. Algara-Siller, O. Lehtinen, F. C. Wang, R. R. Nair, U. Kaiser, H. A. Wu, A. K. Geim, and I. V. Grigorieva, Nature (London) 519, 443 (2015).

[9] K. S. Novoselov, A. K. Geim, S. V. Morozov, D. Jiang, Y. Zhang, S. V. Dubonos, I. V. Grigorieva, and A. A. Firsov, Science 306, 666 (2004).

[10] T. Bjorkman, A. Gulans, A. V. Krasheninnikov, and R. M. Nieminen, Phys. Rev. Lett. 108, 235502 (2012).

[11] S. P. Koenig, N. G. Boddeti, M. L. Dunn, and J. S. Bunch, Nat. Nanotechnol. 6, 543 (2011).
[12] A. C. T. van Duin, S. Dasgupta, F. Lorant, and W. A. Goddard, J. Phys. Chem. A 105, 9396 (2001); A. C. T. van Duin and J. S. S. Damste, Org. Geochem. 34, 515 (2003).

[13] S. Plimpton, J. Comput. Phys. 117, 1 (1995).

[14] K. Chenoweth, A. C. T. van Duin, and W. A. Goddard, J. Phys. Chem. A 112, 1040 (2008).

[15] J. L. Abascal and C. Vega, J. Chem. Phys. 123, 234505 (2005).

[16] We emphasize that flat ice with a perfect square lattice cannot be formed between two graphene layers because the two electrons on the $\mathrm{O}$ atom form two covalent bonds with the $\mathrm{H}$ atoms while the four others make two $\mathrm{H}$ bonds with the neighbor waters (this is responsible for the asymmetry), i.e., the H-O-H angle cannot be $90^{\circ}$.

[17] G. Algara-Siller, O. Lehtinen, F. C. Wang, R. R. Nair, U. Kaiser, H. A. Wu, A. K. Geim, and I. V. Grigorieva (private communications).

[18] M. Chaplin, arXiv:0706.1355.

[19] M. Neek-Amal, P. Xu, D. Qi, P. M. Thibado, L. O. Nyakiti, V. D. Wheeler, R. L. Myers-Ward, C. R. Eddy, Jr., D. K. Gaskill, and F. M. Peeters, Phys. Rev. B 90, 064101 (2014).

[20] W.-H. Zhao, J. Bai, L.-F. Yuan, J. Yang, and X. C. Zeng, Chem. Sci. 5, 1757 (2014).

[21] L. Pauling, J. Am. Chem. Soc. 57, 2680 (1935). 